\title{
OTUB1-mediated deubiquitination of FOXM1 up-regulates ECT-2 to promote tumor progression in renal cell carcinoma
}

\author{
Kai Zhou ${ }^{1 \dagger}$, Haixing Mai ${ }^{2 \dagger}$, Song Zheng ${ }^{1 *}$, Weizhong Cai ${ }^{1}$, Xu Yang ${ }^{1}$, Zhenlin Chen ${ }^{1}$ and Bin Zhan ${ }^{1}$
}

\begin{abstract}
Background: OTUB1 (ovarian tumor domain protease domain-containing ubiquitin aldehyde-binding proteins)mediated deubiquitination of FOXM1 (Forkhead box M1) participates in carcinogenesis of various tumors. We aim to investigate the effect and mechanism of OTUB1/FOXM1 on RCC (renal cell carcinoma) progression. Expression levels of OTUB1 in RCC tissues and cell lines were examined by qRT-PCR (quantitative real-time polymerase chain reaction) and immunohistochemistry. Cell proliferation was measured with CCK8 (Cell Counting Kit-8) and colony formation assays. Wound healing and transwell assays were used to determine cell migration and invasion, respectively. The effect of OTUB1 on FOXM1 ubiquitination was examined by Immunoprecipitation. Western blot was used to uncover the underlying mechanism. In vivo subcutaneous xenotransplanted tumor model combined with immunohistochemistry and western blot were used to examine the tumorigenic function of OTUB1.

Results: OTUB1 was up-regulated in RCC tissues and cell lines, and was associated with poor prognosis of RCC patients. Knockdown of OTUB1 inhibited cell viability and proliferation, as well as migration and invasion of RCC cells. Mechanistically, knockdown of OTUB1 down-regulated FOXM1 expression by promoting its ubiquitination. Downregulation of FOXM1 inhibited ECT2 (epithelial cell transforming 2)-mediated Rho signaling. Moreover, the inhibition of RCC progression caused by OTUB1 knockdown was reversed by FOXM1 over-expression. In vivo subcutaneous xenotransplanted tumor model also revealed that knockdown of OTUB1 could suppress in vivo RCC growth via downregulation of FOXM1-mediated ECT2 expression.
\end{abstract}

Conclusions: OTUB1-mediated deubiquitination of FOXM1 up-regulates ECT-2 to promote tumor progression in RCC, providing a new potential therapeutic target for RCC treatment.

Keywords: OTUB1, FOXM1, ECT-2, Renal cell carcinoma, Progression

\section{Background}

Renal cell carcinoma (RCC) accounts for about 3\% of all tumors with mortality rate as high as $40 \%[1,2]$. With steadily increasing incidence [3], there is urgent need to find novel targets for diagnosis and treatment of RCC. Although current treatments for RCC such as surgical

\footnotetext{
*Correspondence: SongZhengdhji@163.com

${ }^{\dagger}$ Kai Zhou and Haixing Mai contributed equally to the work

${ }^{1}$ Department of Urology, Fujian Medical University Union Hospital, 29 Xinquan Road, Fuzhou 350001, Fujian, China

Full list of author information is available at the end of the article
}

resection or drug targeted therapies have improved tremendously, the lack of effective early diagnostic biomarkers reduces overall survival rates [4]. Moreover, due to high invasiveness and relapse rate, the mortality of RCC appears to be increasing rapidly in the past decade [2]. Therefore, identification of new sensitive diagnostic biomarkers and investigation of the underlying molecular mechanism of new therapeutic targets possess great clinical significance for improving survival rate of RCC patients.

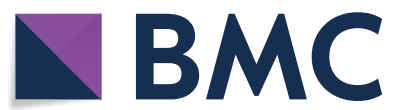

(c) The Author(s) 2020. This article is licensed under a Creative Commons Attribution 4.0 International License, which permits use, sharing, adaptation, distribution and reproduction in any medium or format, as long as you give appropriate credit to the original author(s) and the source, provide a link to the Creative Commons licence, and indicate if changes were made. The images or other third party material in this article are included in the article's Creative Commons licence, unless indicated otherwise in a credit line to the material. If material is not included in the article's Creative Commons licence and your intended use is not permitted by statutory regulation or exceeds the permitted use, you will need to obtain permission directly from the copyright holder. To view a copy of this licence, visit http://creativeco mmons.org/licenses/by/4.0/. The Creative Commons Public Domain Dedication waiver (http://creativecommons.org/publicdomain/ zero/1.0/) applies to the data made available in this article, unless otherwise stated in a credit line to the data. 
Ubiquitination is a post-translational modification via attachment of ubiquitin on lysine residues of the targets [5]. Deubiquitinating enzymes (DUBs) are cysteine proteases that remove ubiquitin from ubiquitinated proteins [6]. DUBs have been widely known as critical regulators in tumor development and progression [7], especially in RCC [8]. Ovarian tumor (OTU)-containing DUBs is one of the members of DUBs [9] and OTUB1 (ovarian tumor domain protease domain-containing ubiquitin aldehydebinding proteins) is a member of OTU domain protease superfamily of DUBs that removes ubiquitin from branched polyubiquitin chains in the target molecules [10]. At present, relevant studies have shown that OTUB1 plays an important regulatory role in various physiological and pathological processes such as DNA damage repair, apoptosis and inflammatory response [11-14]. Recently, the role of OTUB1 on tumorigenesis has been the focus of functional research. Studies have shown that OTUB1 is closely related to the occurrence and development of hepatocellular carcinoma [15], colorectal cancer [16], esophageal squamous cell carcinoma [17], prostate cancer [18], gastric cancer [19] and lung cancer [20]. However, the regulation ability and underlying mechanism of OTUB1 on RCC have not been reported yet.

FOXM1 (Forkhead box M1) functions as a transcriptional factor to regulate expression of proliferation-associated genes and participates in DNA replication and mitosis [21]. FOXM1 has been shown to regulate cell cycle during progression of prostate cancer [22], breast cancer [23], colorectal cancer [24] and RCC [25]. More interestingly, OTUB1 was shown to promote deubiquitination of FOXM1 in breast cancer [26] and ovarian cancer [27] to facilitate tumor progression. Therefore, we hypothesized that OTUB1-mediated deubiquitination of FOXM1 might also participate in RCC progression. We investigated the effect of OTUB1/FOXM1 axis on RCC progression and uncovered the underlying mechanism. Our study may serve as a foundation for the development of novel RCC therapy.

\section{Results}

\section{OTUB1 was elevated in RCC tissues and cell lines}

To explore the correlation between OTUB1 and RCC, we analyzed the expression level of OTUB1 in RCC tissues and cell lines. Using qRT-PCR analysis, we found that OTUB1 was highly expressed in RCC tumor tissues compared to adjacent non-cancer specimens (Fig. 1a). Moreover, immunohistochemistry showed that OTUB1 expression was positively correlated with the TNM stage of RCC (Fig. 1b), suggesting that OTUB1 may contribute to RCC progression. Further analysis of correlation between OTUB1 and clinic pathologic characteristics of RCC patients indicated that among the 67 patients, high expression of OTUB1 $(\mathrm{N}=34)$ was significantly correlated with $\mathrm{T}$ stage $(P=0.007), \mathrm{N}$ stage $(P=0.026)$ and $\mathrm{M}$ stage $(P=0.019)$ (Table 1$)$. Moreover, other clinical features such as histological grade $(P<0.001)$ and TNM stage $(P=0.006)$ were dramatically correlated with high OTUB1 expression, in consistent with immunohistochemistry analysis. However, gender $(P=0.365)$ and age $(P=0.393)$ showed no significant correlation with OTUB1 expression (Table 1). Elevation of OTUB1 was associated with poor prognosis of RCC, suggesting a potential ability of OTUB1 to serve as a prognostic biomarker for RCC. Consistent with expression in RCC tissues, OTUB1 was also elevated in RCC cell lines (Caki-1, ACHN, A-498 and 786-O) compare to HK2 and HUVEC (Fig. 1c, d). Caki-1 and 786-O cells with higher expression of OTUB1 were selected for the following functional assays.

\section{Knockdown of OTUB1 suppressed cell proliferation, migration and invasion of RCC}

Loss-of function assays were conducted to determine the effects of OTUB1 on RCC progression. Two siRNAs targeting OTUB1 (siOTUB1 \#1 and \#2) were designed. The knockdown efficiency was confirmed in Additional file 1: Figure S1A. SiOTUB1 \#1 induced stronger downregulation of OTUB1. Therefore we selected SiOTUB1 $\# 1$ for the functional assays and renamed it as siOTUB1. The knockdown efficiency of siOTUB1 in Caki-1 and 786-O cells was confirmed using qRT-PCR in Fig. 2a. Data from CCK8 (Fig. 2b) and colony formation assay (Fig. 2c) indicated that knockdown of OTUB1 decreased cell viability and inhibited cell proliferation of RCC cells. Moreover, knockdown of OTUB1 suppressed cell migration (Fig. 2d) and invasion (Fig. 2e) of RCC, suggesting that OTUB1 might contribute to cell proliferation and malignant phenotypes of RCC. Furthermore, knockdown of OTUB1 via siOTUB1 \#2 also suppressed cell viability Additional file 1: Figure S1B), proliferation (Additional file 1: Figure S1C) and migration (Additional file 1: Figure S1D) of RCC.

\section{OTUB1 suppressed ubiquitination of FOXM1 in RCC}

OTUB1-mediated deubiquitination of FOXM1 was then investigated in RCC. Knockdown of OTUB1 had no significant effect on FOXM1 mRNA expression (Fig. 3a), while decreased FOXM1 protein expression in Caki-1 and 786-O cells (Fig. 3b). In vivo ubiquitination assay showed that OTUB1 knockdown drastically promoted ubiquitination of FOXM1 (Fig. 3c). We then applied cycloheximide (CHX), a protein synthesis inhibitor in eukaryotic cells, in Caki-1 and 786-O cells transfected with siOTUB1. The result revealed that CHX treatment promoted the decrease of FOXM1 protein, 

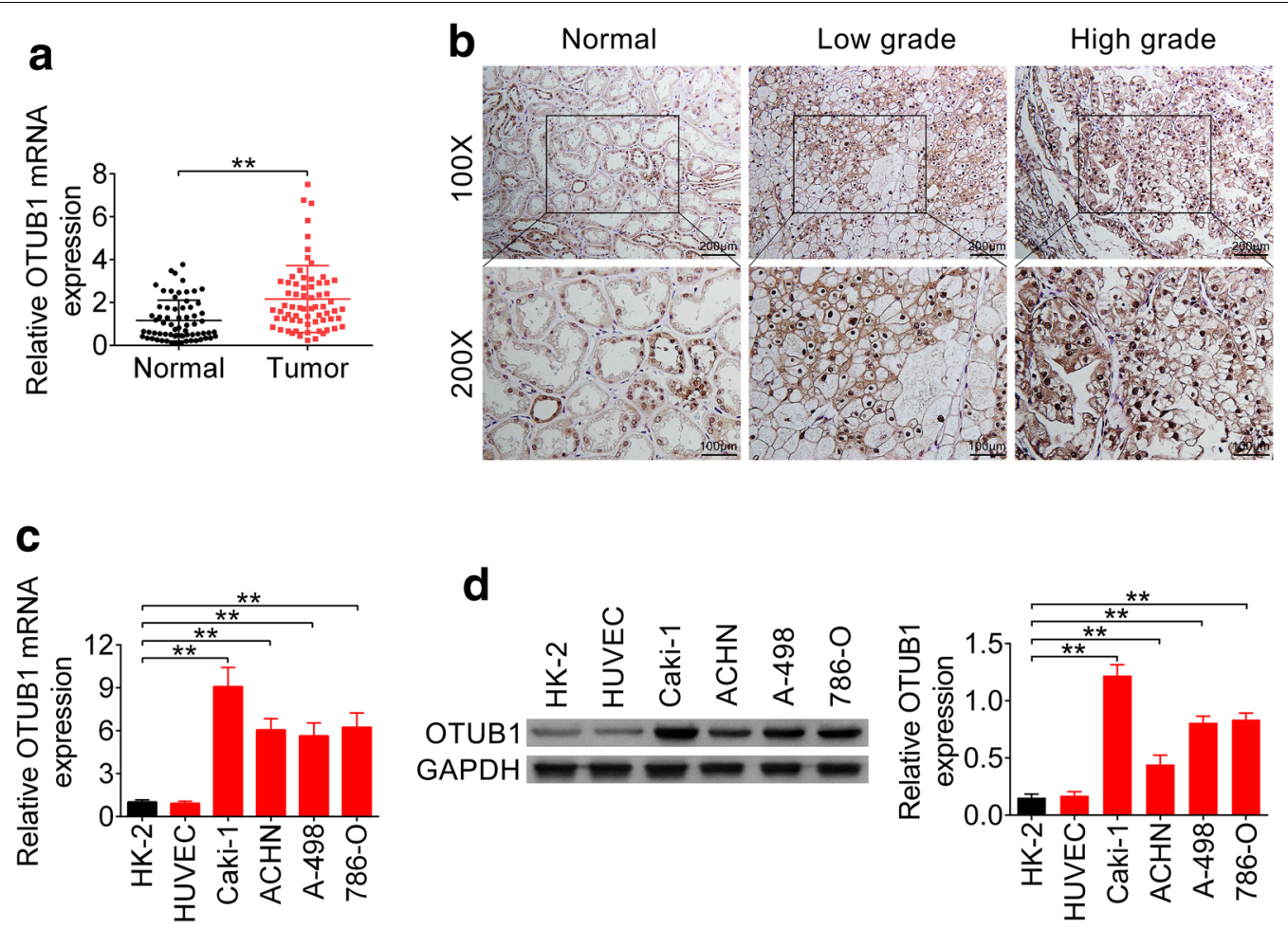

Fig. 1 OTUB1 was elevated in RCC tissues and cell lines. a The expression levels of OTUB1 in RCC tissues and adjacent noncancer tissues were detected by qRT-PCR $(\mathrm{N}=67) .{ }^{* *}$ represents Tumor vs. Normal tissues, $P<0.01$. b Immunohistochemistry analysis of adjacent noncancer tissues and low grade (I+II) or high grade (III + IV) RCC tissues. c The expression levels of OTUB1 in RCC cell lines (Caki-1, ACHN, A-498 and 786-O), HK2 and HUVEC were detected by qRT-PCR ( $N=67)$. ** represents RCC cell lines vs. HK2, $P<0.01$. d The expression levels of OTUB1 in RCC cell lines (Caki-1, ACHN, A-498 and 786-O), HK2 and HUVEC were detected by western blot. ${ }^{* *}$ represents RCC cell lines vs. HK2, $P<0.01$

and the decrease rate of FOXM1 was increased in cells transfected with siOTUB1 (Fig. 3d), suggesting that OTUB1 knockdown suppressed the stability of FOXM1. In addition, the stability of FOXM1 was restored in cells transfected with siNC or siOTUB1 under treatment of proteasome inhibitor MG132 (Fig. 3e), showing that FOXM1 protein was decreased by siOTUB1 in a proteasome-dependent manner. Taken together, these results revealed that OTUB1 suppressed ubiquitination of FOXM1 in RCC.

\section{FOXM1 regulated ECT2-rho signaling}

The downstream target for FOXM1 in RCC was then determined via loss-of function assay. Two siRNAs targeting FOXM1 (siFOXM1 \#1 and \#2) were designed to knock down expression of FOXM1 and both of them efficiently reduced FOXM1 protein expression (Additional file 1: Figure S1E). SiFOXM1 \#1 was named as siFOXM1 and selected for the subsequently functional assays. Knockdown of FOXM1 by siFOXM1 at mRNA level was confirmed in Fig. 4a. Western blot analysis indicated that Caki-1 and 786-O cells transfected with siFOXM1 decreased ECT2 expression compared to cells transfected with siNC (Fig. 4b). Rho signaling, controlled by ECT 2 and fundamental for cell migration and invasion, was then investigated. Proteins involved in Rho signaling, Rho and Rac1, were not altered by knockdown of FOXM1 (Fig. 4b). However, the GTP-loaded active Rho and Rac1 were decreased in cells transfected with siFOXM1 (Fig. 4b), suggesting that FOXM1 regulated ECT2-Rho Signaling to participate in RCC migration and invasion. Moreover, siFOXM1 \#2 also decreased protein expression of FOXM1, ECT2, GTP-Rho and GTP-Rac1 (Additional file 1: Figure S1E).

\section{Inhibition ability of OTUB1 knockdown on RCC progression} was reversed by FOXM1 over-expression

To establish whether FOXM1 is required for OTUB1mediated RCC progression, Caki-1 cells were co-transfected with siOTUB1 and pcDNA 3.1-FOXM1. FOXM1 was decreased in cells transfected with siOTUB1. Cotransfection of siOTUB1 and pcDNA 3.1-FOXM1 reversed the decrease of FOXM1 (Fig. 5a). Colony formation assay showed that the inhibition ability of siOTUB1 on cell proliferation of RCC was reversed by co-transfection of pcDNA 3.1-FOXM1 (Fig. 5b). Moreover, data 


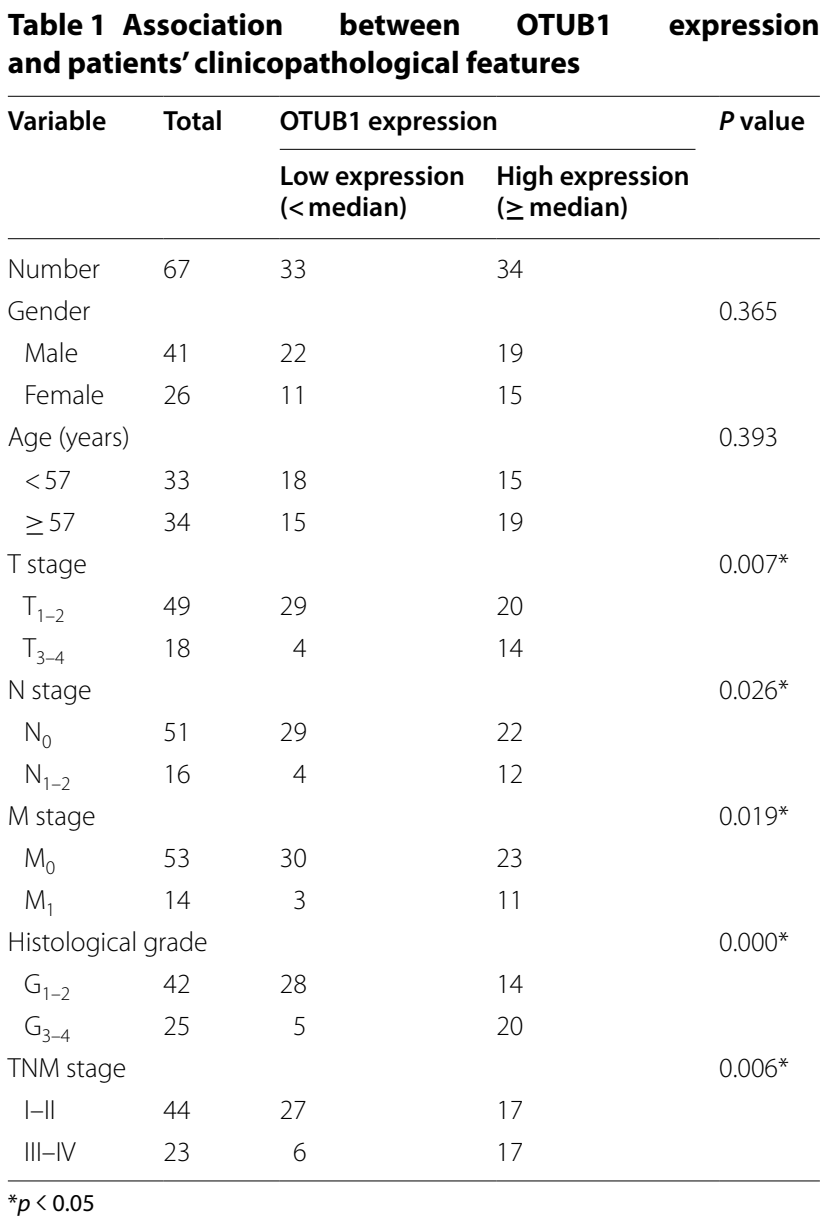

from cell migration (Fig. 5c) and invasion (Fig. 5d) analysis indicated that the suppression abilities of siOTUB1 on cell migration and invasion were also suppressed in cells co-transfected with siOTUB1 and pcDNA 3.1-FOXM1. All these results indicated that inhibition ability of OTUB1 knockdown on RCC progression was reversed by FOXM1 over-expression, confirming the role of OTUB1/ FOXM1 axis on the regulation of RCC progression.

\section{OTUB1 knockdown inhibited in vivo RCC tumor growth}

The in vivo xenograft model via inoculation of AdshOTUB1 into nude mice was constructed to investigate clinical application of OTUB1 knockdown in RCC. Down-regulation of OTUB1 via Ad-shOTUB1 was confirmed in Fig. 6a. Moreover, the injection of Ad-shOTUB1 inhibited tumor growth (Fig. 6b), as shown by decrease of tumor weight and volume. Furtherly, proteins expression of OTUB1, FOXM1, ECT2, GTP-Rho and GTP-Rac1 were all decreased by Ad-shOTUB1 (Fig. 6c), and immunohistochemistry also indicated the downregulation of OTUB1, FOXM1, ECT2 and Ki67 in tissues of mice injected with Ad-shOTUB1 (Fig. 6d). However, total protein level of Rho and Rac1 was not affected by Ad-shOTUB1 (Additional file 1: Figure S1F). These results suggested that OTUB1 knockdown inhibited xenograft tumor growth via regulation of FOXM1-mediated ECT-Rho signaling.

\section{Discussion}

OTUB1, as a member of DUBs, regulates ubiquitination and stabilization of tumorigenesis associated proteins such as p53 [28], estrogen receptor $\alpha$ [29], and SMAD2/3 to participate in tumor progression. Moreover, the tumorigenesis associated proteins p53 [30], estrogen receptor $\alpha$ [31] and SMAD2/3 [32] further regulate FOXM1 expression in various tumors, suggesting the critical role of OTUB1/FOXM1 axis in promotion of tumor progression. Considering that FOXM1 is upregulated in many tumor types [33], and FOXM1 participates in RCC progression [34-36], OTUB1/FOXM1 axis may have broad role in tumor progression across multiple tumor types, especially in RCC. Here our study showed, for the first time, that OTUB1 catalyzed deubiquitination and stabilization of FOXM1 to promote RCC progression.

An elevation of OTUB1 expression was firstly found in the present study and shown to be associated with poor prognosis of RCC, suggesting a potential ability of OTUB1 as a prognostic biomarker for RCC. However, due to the small sample size of current clinical analysis $(\mathrm{N}=67)$ between OTUB1 and RCC, the association of OTUB1 expression level with other clinicopathological features of RCC patients may not be precise enough. A larger patient cohort is needed to strengthen the clinical significance of OTUB1 in RCC patients.

Inhibition of DUBs has been shown to affect the ubiquitination and stabilization of DUB-regulated oncoproteins [37], thus leading to tumor growth inhibition [38]. Therefore, DUBs inhibition has been regarded as a novel potential cancer therapeutic strategy. In line with the clinical results of OTUB1 in RCC, in vitro functional assays revealed that knockdown of OTUB1 inhibited cell proliferation, migration and invasion of RCC cells. Moreover, in vivo subcutaneous xenotransplanted tumor model also indicated that knockdown of OTUB1 could suppress in vivo tumorigenic ability of RCC. In conclusion, OTUB1 may not only function as a potential biomarker for RCC diagnosis, but also serve as a potential novel target for RCC therapy.

The underlying mechanism involved in the regulation of RCC progression via OTUB1 was then investigated in the present study. OTUB1 was reported to inhibit the ubiquitination of FOXM1 in ovarian cancer [27] and breast cancer [26]. In line with these studies, our results showed that knockdown of OTUB1 promoted the ubiquitination of FOXM1 in RCC, and the reduction of FOXM1 by 


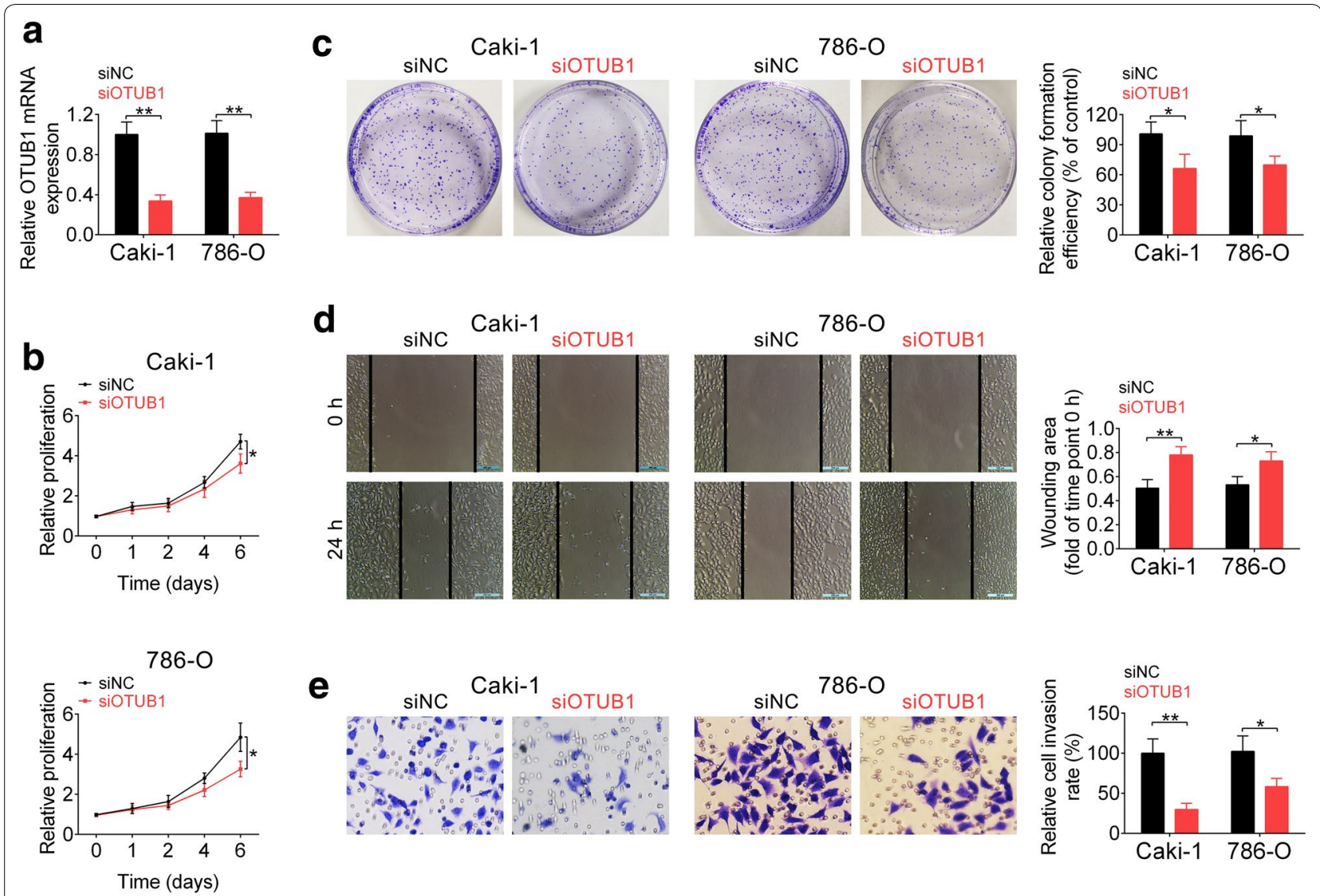

Fig. 2 Knockdown of OTUB1 suppressed cell proliferation, migration and invasion of RCC. a Knockdown efficiency of siOTUB1 in Caki-1 and 786-O cells as measured by qRT-PCR. ${ }^{* *}$ represents siOTUB1 vs. siNC, $p<0.01$. b The effect of OTUB1 knockdown on cell viability of Caki-1 and 786-O cells. ${ }^{*}$ represents siOTUB1 vs. siNC, $p<0.05$. c The effect of OTUB1 knockdown on cell proliferation of Caki-1 and 786-O cells. ${ }^{*}$ represents siOTUB1 vs. siNC, $p<0.05$. d The effect of OTUB1 knockdown on cell migration of Caki-1 and 786-O cells. ${ }^{*}$, ** represents siOTUB1 vs. siNC, $p<0.05, p<0.01$. e The effect of OTUB1 knockdown on cell invasion of Caki-1 and 786-O cells. * ${ }^{* *}$ represents siOTUB1 vs. siNC, $p<0.05, p<0.01$

protein synthesis inhibitor $(\mathrm{CHX})$ treatment was accelerated via siOTUB1. Moreover, OTUB1 generally restricts the ubiquitination of target proteins in proteasomedependent manner [39]. Our results also showed that proteasome inhibitor (MG132) treatment increased the stability of FOXM1 in RCC. Furthermore, proteasomedependent degradation is always associated with proteins with Lys48-linked polyubiquitin chains [40], and OTUB1 prefers target proteins with polyubiquitin chains joined by Lys48 [41], and catalyzes cleavage of the Lys48-linked polyubiquitin chains from FOXM1 [26, 27]. The Lys48linked polyubiquitin chains deubiquitination of FOXM1 via OTUB1 in RCC needs to be further investigated.

Functional assays indicated that inhibition ability of OTUB1 knockdown on RCC progression was reversed by FOXM1 over-expression, suggesting OTUB1/FOXM1 axis plays a role on the regulation of RCC progression. Although FOXM1 has been shown to participate in RCC progression [25, 34-36], the downstream target is yet to be reported. Here, we found out that ECT2-Rho signaling was involved in the regulation of OTUB1/FOXM1 in RCC. Polo-like kinase 1 (PLK1) phosphorylates FOXM1 to regulate $\mathrm{G} 2 / \mathrm{M}$ transition of mitotic cell cycle in RCC [34]. Moreover, PLK1 also phosphorylates ECT2 to regulate G2/M transition of mitotic cell cycle [42]. ECT2 is over-expressed in various tumors and functions as an oncoprotein to promote tumor progression. Knockdown of ECT2 inhibited tumor cell proliferation, migration and invasion [43]. The present study showed that knockdown of FOXM1 decreased ECT2 expression in RCC, thus may inhibit RCC progression. The oncogenic activity of ECT2 works through Rho signaling in breast cancer [44] and hepatocellular carcinoma [45] by transforming inactive GDP-loaded state of Rho to active GTP-loaded state. Our results showed that knockdown of FOXM1 decreased active GTP-loaded state of Rho (GTP-Rho and GTP-Rac1) in RCC, therefore inactivating ECT2Rho signaling to suppress tumor growth. Moreover, the 
a
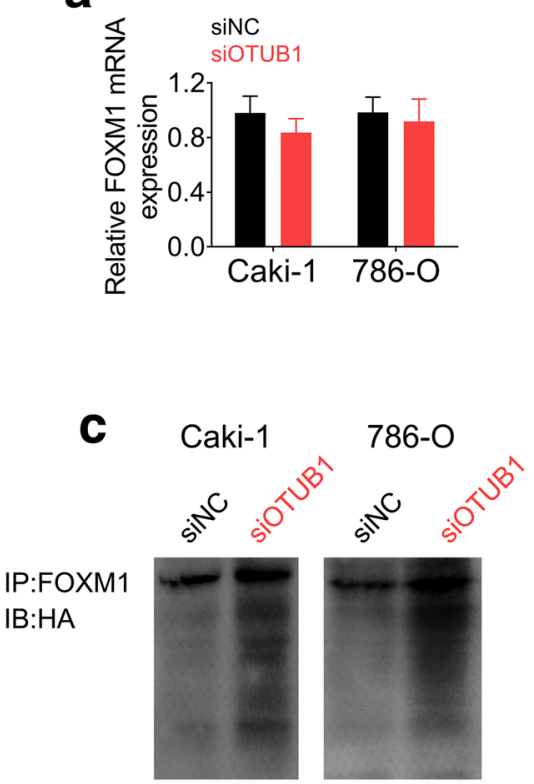

IB: FOXM1

GAPDH

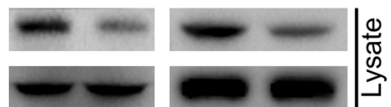

b

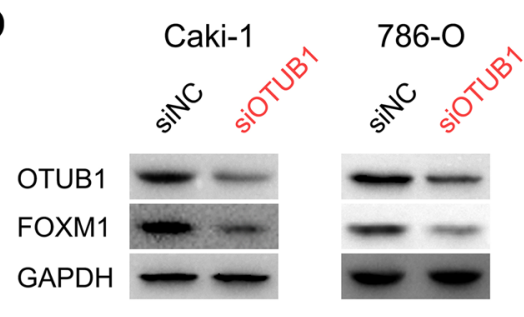

d

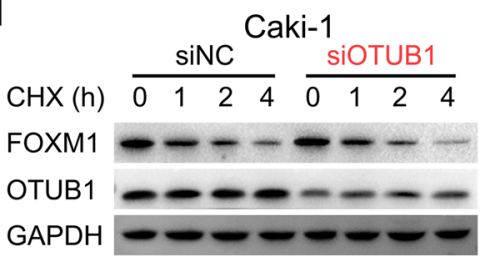

$\mathbf{e}$

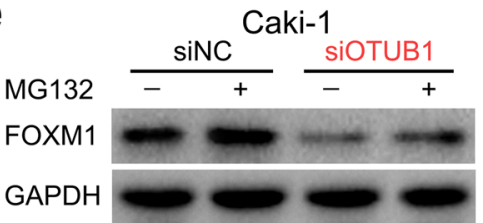

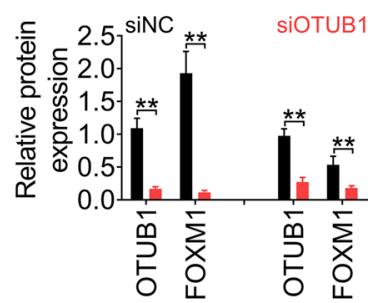

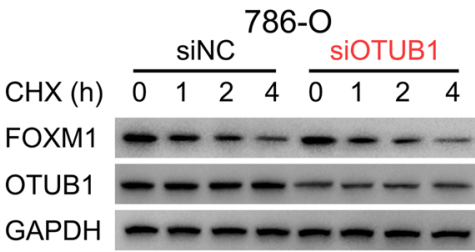

MG132 $\frac{\text { sinC }^{786-O}}{-} \frac{\text { siOTUB1 }}{-}$

FOXM1

GAPDH

Fig. 3 OTUB1 suppressed ubiquitination of FOXM1 in RCC. a The effect of OTUB1 knockdown on FOXM1 mRNA expression in Caki-1 and 786-O cells. $\mathbf{b}$ The effect of OTUB1 knockdown on FOXM1 protein expression in Caki-1 and 786-O cells. ${ }^{* *}$ represents siOTUB1 vs. siNC, $p<0.01$. $\mathbf{c}$ The effect of OTUB1 knockdown on FOXM1 ubiquitination in Caki-1 and 786-O cells. $\mathbf{d}$ The effect of OTUB1 knockdown on FOXM1 protein expression in Caki-1 and 786-O cells under CHX treatment. e The effect of OTUB1 knockdown on FOXM1 protein expression in Caki-1 and 786-O cells under MG132 treatment

oncogenic activity of ECT2 also dependents on protein kinase C iota-mediated phosphorylation [46], the effect of OTUB1/FOXM1 axis on regulation of protein kinase $\mathrm{C}$ iota needs to be further investigated.

\section{Conclusion}

OTUB1 regulated ubiquitination and stabilization of FOXM1, and OTUB1/FOXM1 axis contributes to RCC tumorigenesis and aggression via ECT2-Rho signaling, suggesting a novel insight into the treatment of RCC.

\section{Methods}

\section{Patient samples and immunohistochemistry}

Surgical cancer or adjacent noncancer specimens from 67 RCC patients were collected at Fujian Medical University Union Hospital. The study was approved by the Ethics Committee of Fujian Medical University Union Hospital, and all the patients signed written informed consent. Paraffined RCC tissues were cut into $4 \mu \mathrm{m}$ thick sections. The sections were then dewaxed and rehydrated. After washing with PBS (Phosphate Buffered Saline), the sections were blocked with $2 \%$ and $0.5 \%$ goat serum in PBS, and then incubated overnight with primary rabbit antibodies against OTUB1, FOXM1, ECT2, Ki67 (Abcam, Cambridge, MA, USA). HRP (horseradish peroxidase, Sigma Aldrich, St. Louis, MO, USA)-conjugated goat anti-rabbit IgG secondary antibody was then added to the sections. The slides were counterstained with hematoxylin and examined under light microscope (Olympus, Tokyo, Japan).

\section{Cell culture}

Human RCC cell lines (Caki-1, ACHN, A-498 and 786O), HK2 (human renal proximal tubular epithelial cell line) and HUVEC (human umbilical vein endothelial cell) were purchased from the Chinese Academy of Sciences (Shanghai, China). All the cell lines were cultured in RPMI-1640 medium (Gibco; Thermo Fisher, Waltham, MA, USA) supplemented with $10 \%$ fetal bovine serum at $37^{\circ} \mathrm{C}$ constant temperature incubator with $5 \% \mathrm{CO}_{2}$.

\section{Cell transfection}

siRNAs targeting OTUB1 or FOXM1 as well as the negative control (siNC) were synthesized by GenePharma 
a

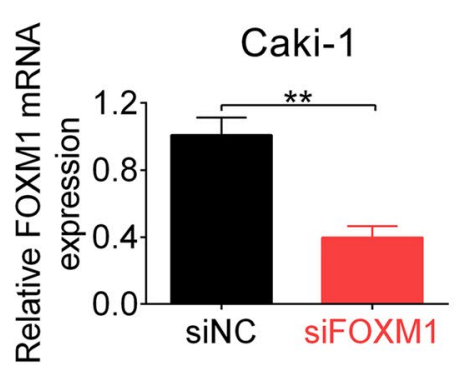

b
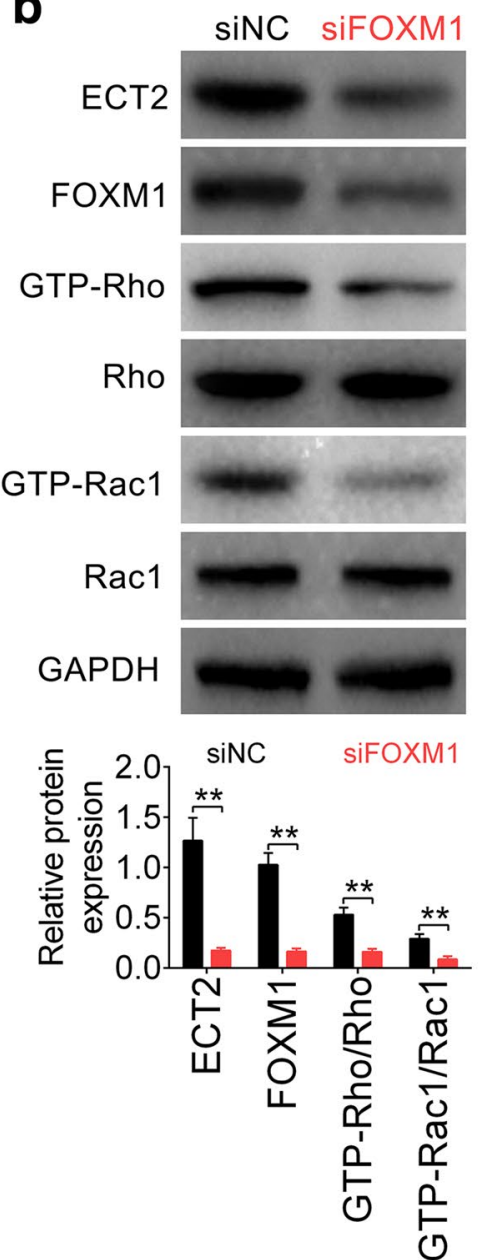

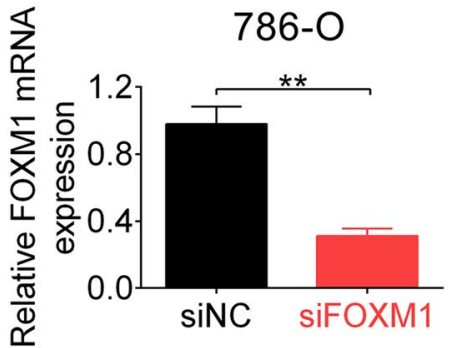

786-O
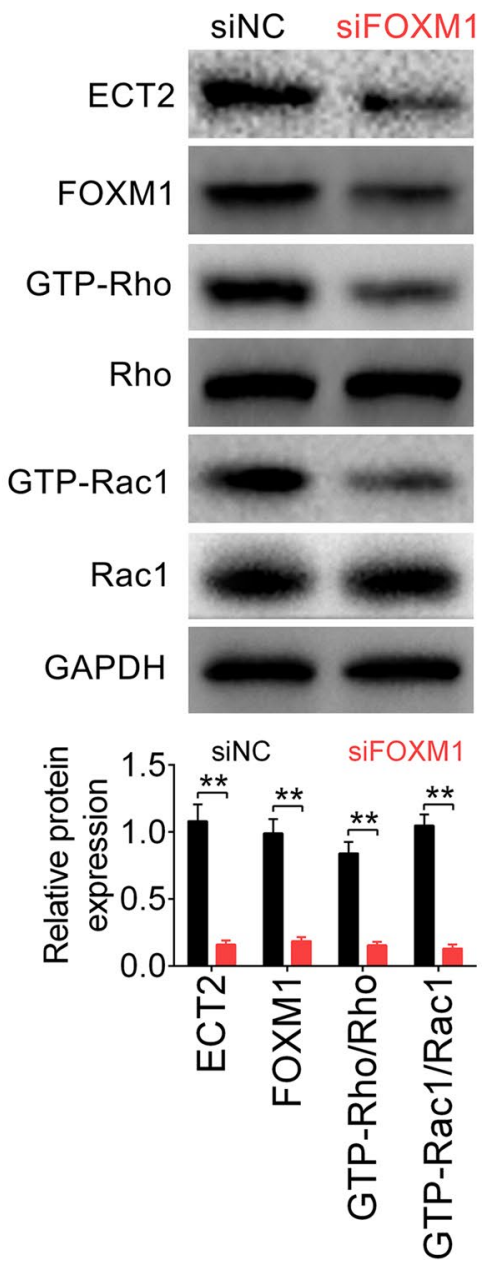

Fig. 4 FOXM1 regulated ECT2-Rho Signaling. a Knockdown efficiency of siFOXM1 in Caki-1 and 786-O cells as measured by qRT-PCR. ** represents siFOXM1 vs. siNC, $p<0.01$. b The effect of FOXM1 knockdown on proteins expression of ECT2, FOXM1, Rho, GTP-Rho, Rac1 and GTP-Rac1 in Caki-1 and 786-O cells. ** represents siFOXM1 vs. siNC, $p<0.01$

(Shanghai, China). pcDNA3.1-FOXM1 and the negative control (pcDNA3.1-NC) were obtained from AxyBio co., LTD (Changsha, China). Caki-1 and 786-O cells with $1 \times 10^{6}$ cells/well were seeded into 12 -well plate and then transfected with siOTUB1, siFOXM1,
siNC, pcDNA3.1-FOXM1 or pcDNA3.1-NC via Lipofectamine ${ }^{\circledR} 3000$ (Thermo Fisher, Waltham, MA, USA). Two days transfection, the cells were collected for the following experiments. 


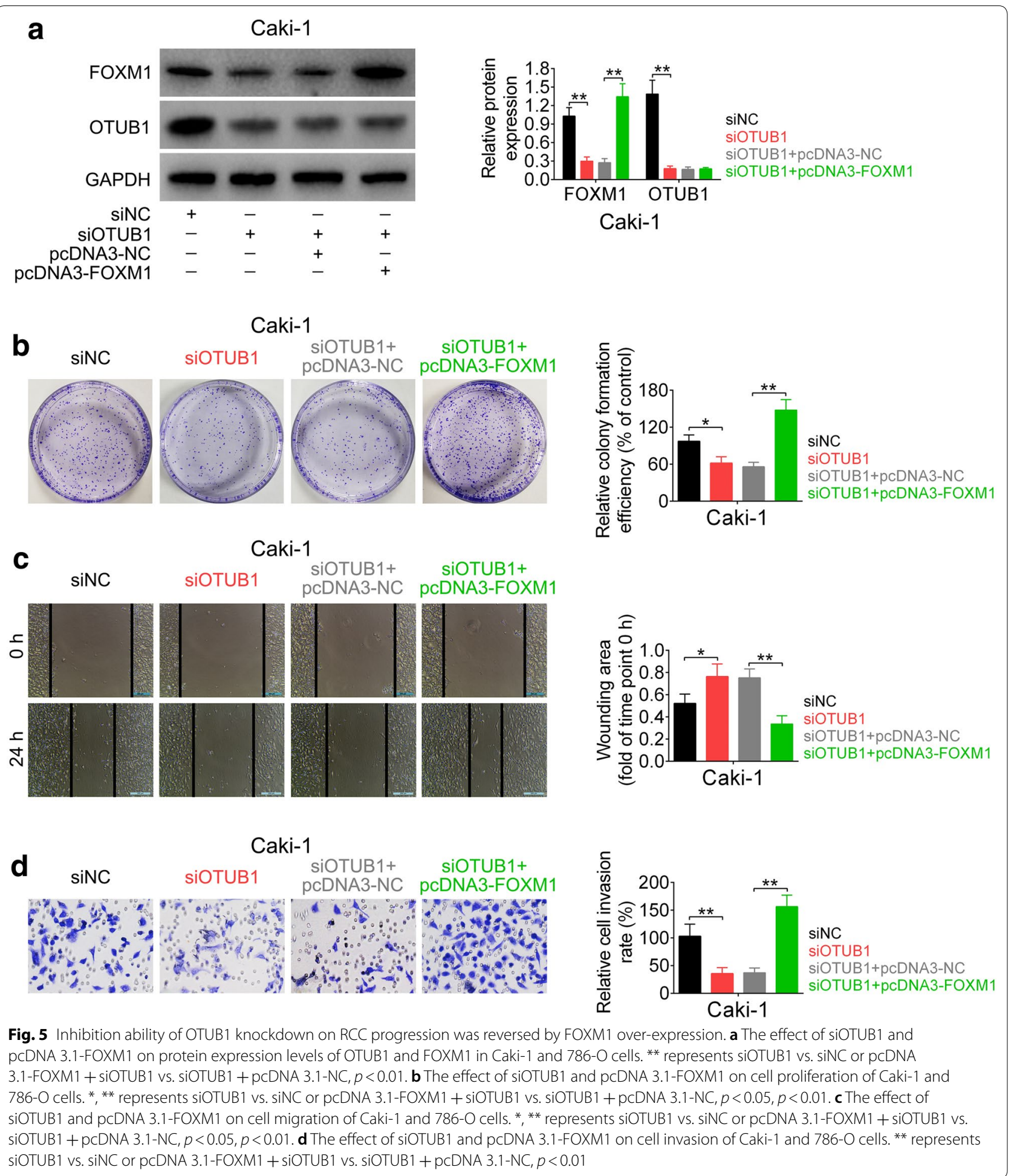


a
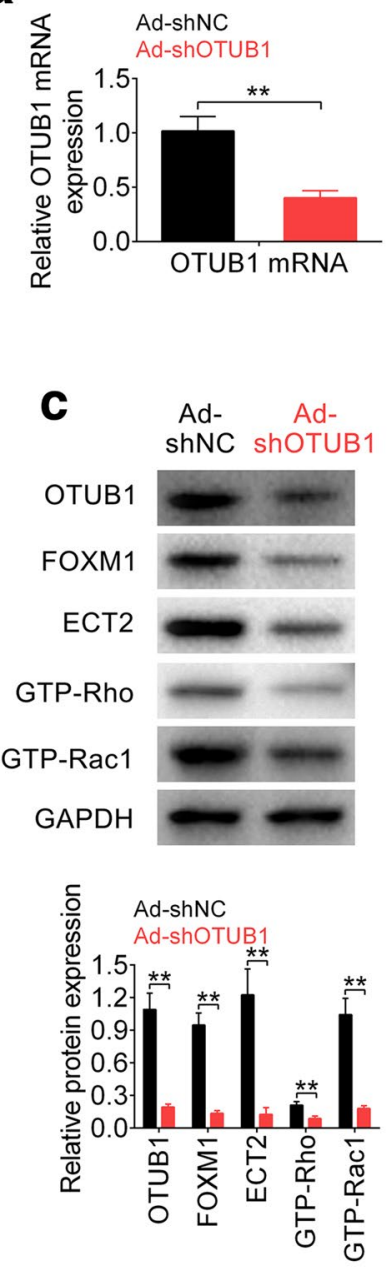
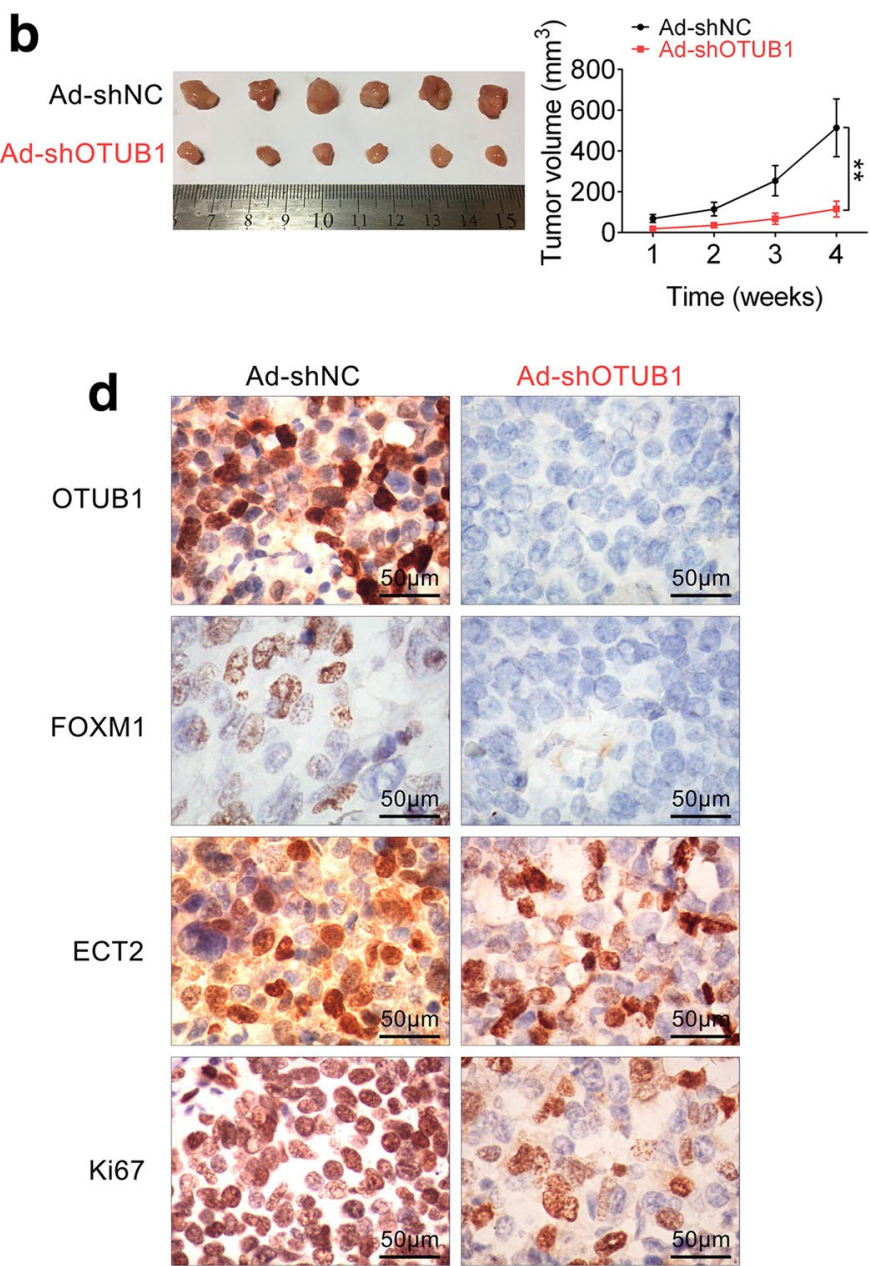

Fig. 6 OTUB1 knockdown inhibited in vivo RCC tumor growth. a Knockdown efficiency of Ad-shOTUB1 in nude mice as measured by qRT-PCR. ** represents Ad-shOTUB1 vs. Ad-shNC, $p<0.01$. b The effect of Ad-shOTUB1 on RCC tumor growth and volume. ** represents Ad-shOTUB1 vs. Ad-shNC, $p<0.01$. c The effect of Ad-shOTUB1 on proteins expression of OTUB1, FOXM1, ECT2, GTP-Rho and GTP-Rac1 in xenograft tumor mice. ** represents Ad-shOTUB1 vs. Ad-shNC, $p<0.01$. d Immunohistochemistry analysis of OTUB1, FOXM1, ECT2 and Ki67 in tissues of mice intratumorally injected with Ad-shOTUB1 or Ad-shNC

\section{Cell proliferation assay}

Caki-1 and 786-O cells with $5 \times 10^{3}$ cells/well were seeded in 96-well plates. At 0, 1, 2, 4, 6 days, $20 \mu \mathrm{L}$ CCK8 solution (Dojindo, Tokyo, Japan) was added into each well and mixed for $3 \mathrm{~h}$. Microplate Autoreader (BioTek, Winooski, VT, USA) was used to measure optical density at $450 \mathrm{~nm}$. For colony formation experiments, Caki-1 and 786-O cells with 200 cells/well were seeded on six-well plate with RPMI 1640 medium. Fourteen days later, the cells were fixed in formalin and stained with crystal violet $(0.1 \%)$. The visible colonies were counted and photographed under light microscope (Olympus).

\section{Wound healing and transwell assay}

For cell migration analysis, Caki-1 and 786-O cells were seeded in 6-well plates. Wound gap in the cell monolayer was generated by scratching with plastic pipette tip. The cells were washed with PBS to remove debris or the detached cells, and cultured in RPMI-1640 for another $48 \mathrm{~h}$ before measuring the wound width. For cell invasion analysis, transfected Caki-1 and 786-O cells were seeded onto the upper wells of chamber (Corning, MA, USA) with the Matrigel-coated membrane (BD Biosciences, Franklin Lakes, NJ, USA) in serum-free RPMI 1640 medium. RPMI 1640 medium with $20 \%$ FBS were added to the lower wells. The medium of upper wells and the 
filters were removed $8 \mathrm{~h}$ later. The invasive cells to the bottom of chambers were fixed with $100 \%$ methanol and then stained with $0.1 \%$ crystal violet $24 \mathrm{~h}$ later, imaged and counted under microscope.

\section{Ubiquitination/deubiquitination and FOXM1 protein turnover assays}

Caki-1 and 786-O cells transfected with siOTUB1 or siNC were firstly treated with $10 \mu \mathrm{M}$ MG132 for $3 \mathrm{~h}$ and then collected and lysed. The lysates were immunoprecipitated by anti-FOXM1 (Abcam) and immunoblotted by anti-ubiquitin (Abcam). For measurement of endogenous FOXM1 turnover rate, Caki-1 and 786-O cells transfected with siOTUB1 or siNC were treated with $80 \mu \mathrm{g} / \mathrm{mL}$ cycloheximide (CHX) (Sigma-Aldrich, St. Louis, MO, USA) for inhibition of protein synthesis. At $0,1,2,4 \mathrm{~h}$, cells were harvested and analyzed by western blot.

\section{Quantitative real-time PCR (qRT-PCR)}

Total RNAs from RCC tissues or cell lines were extracted via RNeasy Mini Kit (Qiagen, Manchester, UK). Complementary DNAs were then generated by PrimeScript RT Reagent (Takara, Shiga, Japan). qRT-PCR was analyzed by ViiA 7 (Applied Biosystems, Austin, TX, USA), and the expression fold changes of indicated genes were compared with GAPDH and calculated VIA using $2^{-\Delta \Delta C t}$ methods. The primer sequences were showed as follows in primer Table 2.

\section{Western blot}

$30 \mu \mathrm{g}$ proteins from RCC tissues or cells were separated by sodium dodecyl sulfate-polyacrylamide gel electrophoresis, and then transferred to nitrocellulose membrane (Millipore, Bedford, MA). The membranes were blocked by $5 \%$ skimmed milk and then incubated overnight with primary antibodies: anti-OTUB1, antiFOXM1 antibodies (1:1500, Abcam), ECT2 (1:2000, Abcam), GTP-Rho, Rho, GTP-Rac1 and Rac1 (1:2500, Abcam), GAPDH (1:3000, Abcam) at $4{ }^{\circ} \mathrm{C}$. Lastly, the immunoreactivities were detected by enhanced

Table 2 Primer

\begin{tabular}{ll}
\hline ID & Sequence $\left(\mathbf{5}^{\prime} \mathbf{-} \mathbf{3}^{\prime}\right)$ \\
\hline GAPDH F & ACCACAGTCCATGCCATCAC \\
GAPDH R & TCCACCACCCTGTTGCTGTA \\
OTUB1 F & ACAGAAGATCAAGGACCTCCA \\
OTUB1 R & CAACTCCTTGCTGTCATCCA \\
FOXM1 F & ATACGTGGATTGAGGACCACT \\
FOXM1 R & TCCAATGTCAAGTAGCGGTTG \\
\hline
\end{tabular}

chemiluminescence (KeyGen, Nanjin, China) after incubating with HRP labeled secondary antibody (1:5000; Abcam).

\section{Mouse xenograft assay}

Twelve four-to-five week old female BALB/c nude mice with 18-20 g were purchased from the Animal Center of Wenzhou Medical University (Wenzhou, China), and then separated into two groups. The experimental procedures were conducted in accordance with the guidelines set out by Ethics Committee of the Fifth Medical Center of PLA General Hospital. Ad-shOUTB1, as well as the negative control (Ad-shNC), were constructed by GenePharma (Shanghai, China). $100 \mu \mathrm{L} 1 \times 10^{9}$ transducing units Ad-shNC or Ad-shOTUB1 were injected into the flank regions of nude mice. Tumors were measured with digital calipers every week and the tumor volume was calculated. Four weeks later, mice were anesthetized with $65 \mathrm{mg}$ per $\mathrm{kg}$ body weight of sodium pentobarbital, and the xenograft tissues were collected for analysis.

\section{Statistical analysis}

The data were shown as mean \pm standard deviation, and the statistics analysis was performed by the SPSS 19.0 (SPSS, Chicago, IL). Student's t text was used to compare the difference between two groups, one-way ANOVA with Turkey's test to compare the difference among multiple groups. $P<0.05$ was considered as statistically significant.

\section{Supplementary information}

Supplementary information accompanies this paper at https://doi. org/10.1186/s13578-020-00408-0.

Additional file 1: Figure S1. A Knockdown efficiency of siOTUB1 \#1 and \#2 in Caki-1 cells, and the effect of OTUB1 knockdown on protein expression of FOXM1. ${ }^{*},{ }^{* *}$ represents siOTUB1 vs. siNC, $P<0.05, P<0.01$. B The effect of OTUB1 knockdown on cell viability of Caki-1 cells. * represents siOTUB1 vs. siNC, $P<0.05$. C The effect of OTUB1 knockdown on cell proliferation of Caki-1 cells. ${ }^{* *}$ represents siOTUB1 vs. siNC, $P<0.01$. D The effect of OTUB1 knockdown on cell migration of Caki-1 cells. ${ }^{* *}$ represents siOTUB1 vs. siNC, $P<0.01$. E The effect of FOXM1 knockdown on protein expression levels of ECT2, FOXM1, GTP-Rho and GTP-Rac1 in Caki-1 cells. ** represents siFOXM1 vs. siNC, $P<0.01$. F The effect of Ad-shOTUB1 on protein expression levels of GTP-Rho, Rho, GTP-Rac1 and Rac1 in xenograft tumor mice. ${ }^{* *}$ represents Ad-shOTUB1 vs. Ad-shNC, $P<0.01$.

\section{Abbreviations}

CCK8: Cell Counting Kit-8; DUBs: Deubiquitinating enzymes; ECT2: Epithelial cell transforming 2; FOXM1: Forkhead box M1.

\section{Acknowledgements \\ Not applicable.}

\section{Authors' contributions}

KZ, HXM and SZ conceived and designed the experiments. WZC and XY analyzed and interpreted the results of the experiments. ZLC and BZ performed the experiments. All authors read and approved the final manuscript. 


\section{Funding}

This work was supported by the Fujian Province "Double High" Special Research Program and National Natural Science Foundation of China (Grant No. 81872090)

\section{Availability of data and materials}

All data generated or analyzed during this study are included in this published article.

\section{Ethics approval and consent to participate}

The animal use protocol listed below has been reviewed and approved by the Animal Ethical and Welfare Committee.Approval No. 2019069D.

\section{Consent for publication}

Not applicable.

\section{Competing interests}

The authors declare that they have no competing interests, and all authors should confirm its accuracy.

\section{Author details}

${ }^{1}$ Department of Urology, Fujian Medical University Union Hospital, 29 Xinquan Road, Fuzhou 350001, Fujian, China. ${ }^{2}$ Department of Urology, Chinese PLA General Hospital, Beijing 100853, China.

Received: 11 December 2019 Accepted: 13 March 2020

Published online: 30 March 2020

\section{References}

1. Sudhakaran S, Bottiglieri T, Tecson KM, Kluger AY, McCullough PA. Alteration of lipid metabolism in chronic kidney disease, the role of novel antihyperlipidemic agents, and future directions. Rev Cardiovasc Med. 2018;19(3):77-88.

2. Bray F, Ferlay J, Soerjomataram I, Siegel RL, Torre LA, Jemal A. Global cancer statistics 2018: GLOBOCAN estimates of incidence and mortality worldwide for 36 cancers in 185 countries. CA Cancer J Clin. 2018:68(6):394-424.

3. Olshan AF, Kuo TM, Meyer AM, Nielsen ME, Purdue MP, Rathmell WK. Racial difference in histologic subtype of renal cell carcinoma. Cancer Med. 2013;2(5):744-9.

4. Siegel RL, Miller KD, Jemal A. Cancer statistics, 2018. CA Cancer J Clin. 2018;68(1):7-30.

5. Wertz IE, Wang X. From discovery to bedside: targeting the ubiquitin system. Cell Chem Biol. 2019:26(2):156-77.

6. Ramanathan HN, Ye Y. Cellular strategies for making monoubiquitin signals. Crit Rev Biochem Mol Biol. 2012;47(1):17-28.

7. McClurg UL, Robson CN. Deubiquitinating enzymes as oncotargets. Oncotarget. 2015:6(12):9657-68.

8. Poondla N, Chandrasekaran AP, Kim KS, Ramakrishna S. Deubiquitinating enzymes as cancer biomarkers: new therapeutic opportunities? BMB Rep. 2019:52(3):181-9.

9. Nijman SM, Luna-Vargas MP, Velds A, Brummelkamp TR, Dirac AM, Sixma TK, et al. A genomic and functional inventory of deubiquitinating enzymes. Cell. 2005;123(5):773-86.

10. Balakirev MY, Tcherniuk SO, Jaquinod M, Chroboczek J. Otubains: a new family of cysteine proteases in the ubiquitin pathway. EMBO Rep. 2003:4(5):517-22.

11. Wiener R, Zhang X, Wang T, Wolberger C. The mechanism of OTUB1mediated inhibition of ubiquitination. Nature. 2012:483(7391):618-22.

12. Juang YC, Landry MC, Sanches M, Vittal V, Leung CC, Ceccarelli DF, et al. OTUB1 co-opts Lys48-linked ubiquitin recognition to suppress E2 enzyme function. Mol Cell. 2012;45(3):384-97.

13. Chen $Y$, Wang YG, Li Y, Sun XX, Dai MS. Otub1 stabilizes MDMX and promotes its proapoptotic function at the mitochondria. Oncotarget. 2017;8(7):11053-62.

14. Goncharov T, Niessen K, de Almagro MC, Izrael-Tomasevic A, Fedorova AV, Varfolomeev E, et al. OTUB1 modulates C-IAP1 stability to regulate signalling pathways. EMBO J. 2013;32(8):1103-14.
15. Ni Q, Chen J, Li X, Xu X, Zhang N, Zhou A, et al. Expression of OTUB1 in hepatocellular carcinoma and its effects on HCC cell migration and invasion. Acta Biochim Biophys Sin. 2017:49(8):680-8.

16. Zhou Y, Wu J, Fu X, Du W, Zhou L, Meng X, et al. OTUB1 promotes metastasis and serves as a marker of poor prognosis in colorectal cancer. Mol Cancer. 2014;13:258.

17. Zhou H, Liu Y, Zhu R, Ding F, Cao X, Lin D, et al. OTUB1 promotes esophageal squamous cell carcinoma metastasis through modulating Snail stability. Oncogene. 2018:37(25):3356-68.

18. Iglesias-Gato D, Chuan YC, Jiang N, Svensson C, Bao J, Paul I, et al. OTUB1 de-ubiquitinating enzyme promotes prostate cancer cell invasion in vitro and tumorigenesis in vivo. Mol Cancer. 2015;14:8.

19. Wang $Y Q$, Zhang QY, Weng WW, Wu Y, Yang YS, Shen C, et al. Upregulation of the non-coding RNA OTUB1-isoform 2 contributes to gastric cancer cell proliferation and invasion and predicts poor gastric cancer prognosis. Int J Biol Sci. 2016;12(5):545-57.

20. Baietti MF, Simicek M, Abbasi Asbagh L, Radaelli E, Lievens S, Crowther J, et al. OTUB1 triggers lung cancer development by inhibiting RAS monoubiquitination. EMBO Mol Med. 2016;8(3):288-303.

21. Laoukili J, Stahl M, Medema RH. FoxM1: at the crossroads of ageing and cancer. Biochem Biophys Acta. 2007;1775(1):92-102.

22. Kim MY, Jung AR, Kim GE, Yang J, Ha US, Hong SH, et al. High FOXM1 expression is a prognostic marker for poor clinical outcomes in prostate cancer. J Cancer. 2019;10(3):749-56.

23. Bektas N, Haaf A, Veeck J, Wild PJ, Luscher-Firzlaff J, Hartmann A, et al. Tight correlation between expression of the Forkhead transcription factor FOXM1 and HER2 in human breast cancer. BMC Cancer. 2008:8:42.

24. Li D, Wei P, Peng Z, Huang C, Tang H, Jia Z, et al. The critical role of dysregulated FOXM1-PLAUR signaling in human colon cancer progression and metastasis. Clin Cancer Res. 2013;19(1):62-72.

25. Wu XR, Chen YH, Liu DM, Sha JJ, Xuan HQ, Bo JJ, et al. Increased expression of forkhead box M1 protein is associated with poor prognosis in clear cell renal cell carcinoma. Med Oncol. 2013;30(1):346.

26. Karunarathna U, Kongsema M, Zona S, Gong C, Cabrera E, Gomes AR, et al. OTUB1 inhibits the ubiquitination and degradation of FOXM1 in breast cancer and epirubicin resistance. Oncogene. 2016;35(11):1433-44.

27. Wang Y, Zhou X, Xu M, Weng W, Zhang Q, Yang Y, et al. OTUB1-catalyzed deubiquitination of FOXM1 facilitates tumor progression and predicts a poor prognosis in ovarian cancer. Oncotarget. 2016;7(24):36681-97.

28. Sun XX, Challagundla KB, Dai MS. Positive regulation of p53 stability and activity by the deubiquitinating enzyme Otubain 1. The EMBO journal. 2012;31(3):576-92.

29. Stanisic V, Malovannaya A, Qin J, Lonard DM, O'Malley BW. OTU Domaincontaining ubiquitin aldehyde-binding protein 1 (OTUB1) deubiquitinates estrogen receptor (ER) alpha and affects ERalpha transcriptional activity. J Biol Chem. 2009;284(24):16135-45.

30. Millour J, de Olano N, Horimoto Y, Monteiro LJ, Langer JK, Aligue R, et al. ATM and p53 regulate FOXM1 expression via E2F in breast cancer epirubicin treatment and resistance. Mol Cancer Ther. 2011;10(6):1046-58.

31. Millour J, Constantinidou D, Stavropoulou AV, Wilson MS, Myatt SS, Kwok $J M$, et al. FOXM1 is a transcriptional target of ERalpha and has a critical role in breast cancer endocrine sensitivity and resistance. Oncogene. 2010;29(20):2983-95.

32. Xue J, Lin X, Chiu WT, Chen YH, Yu G, Liu M, et al. Sustained activation of SMAD3/SMAD4 by FOXM1 promotes TGF-beta-dependent cancer metastasis. J Clin Investig. 2014;124(2):564-79.

33. Alvarez-Fernandez M, Medema RH. Novel functions of FoxM1: from molecular mechanisms to cancer therapy. Front Oncol. 2013;3:30.

34. Zhang Z, Zhang G, Kong C. FOXM1 participates in PLK1-regulated cell cycle progression in renal cell cancer cells. Oncol Lett. 2016;11(4):2685-91.

35. Liu F, Li N, Liu Y, Zhang J, Zhang J, Wang Z. Homeodomain interacting protein kinase-2 phosphorylates FOXM1 and promotes FOXM1mediated tumor growth in renal cell carcinoma. J Cell Biochem. 2019;120(6):10391-401.

36. Xue YJ, Xiao RH, Long DZ, Zou XF, Wang XN, Zhang GX, et al. Overexpression of FoxM1 is associated with tumor progression in patients with clear cell renal cell carcinoma. J Transl Med. 2012;10:200.

37. Buckley DL, Crews CM. Small-molecule control of intracellular protein levels through modulation of the ubiquitin proteasome system. Angew Chem. 2014:53(9):2312-30. 
38. D'Arcy $P$, Wang $X$, Linder $S$. Deubiquitinase inhibition as a cancer therapeutic strategy. Pharmacol Ther. 2015;147:32-54.

39. Nakada S, Tai I, Panier S, Al-Hakim A, lemura S, Juang YC, et al. Non-canonical inhibition of DNA damage-dependent ubiquitination by OTUB1. Nature. 2010;466(7309):941-6.

40. Komander D, Rape M. The ubiquitin code. Annu Rev Biochem. 2012:81:203-29.

41. Mevissen TE, Hospenthal MK, Geurink PP, Elliott PR, Akutsu M, Arnaudo N, et al. OTU deubiquitinases reveal mechanisms of linkage specificity and enable ubiquitin chain restriction analysis. Cell. 2013;154(1):169-84.

42. Su KC, Takaki T, Petronczki M. Targeting of the RhoGEF Ect2 to the equatorial membrane controls cleavage furrow formation during cytokinesis. Dev Cell. 2011;21(6):1104-15.

43. Fields $A P$, Justilien $V$. The guanine nucleotide exchange factor (GEF) Ect2 is an oncogene in human cancer. Adv Enzyme Regul. 2010;50(1):190-200.
44. Mansour M, Haupt S, Chan AL, Godde N, Rizzitelli A, Loi S, et al. The E3-ligase E6AP represses breast cancer metastasis via regulation of ECT2Rho signaling. Cancer Res. 2016;76(14):4236-48.

45. Chen J, Xia H, Zhang X, Karthik S, Pratap SV, Ooi LL, et al. ECT2 regulates the Rho/ERK signalling axis to promote early recurrence in human hepatocellular carcinoma. J Hepatol. 2015;62(6):1287-95.

46. Justilien V, Jameison L, Der CJ, Rossman KL, Fields AP. Oncogenic activity of Ect2 is regulated through protein kinase C iota-mediated phosphorylation. J Biol Chem. 2011;286(10):8149-57.

\section{Publisher's Note}

Springer Nature remains neutral with regard to jurisdictional claims in published maps and institutional affiliations.
Ready to submit your research? Choose BMC and benefit from:

- fast, convenient online submission

- thorough peer review by experienced researchers in your field

- rapid publication on acceptance

- support for research data, including large and complex data types

- gold Open Access which fosters wider collaboration and increased citations

- maximum visibility for your research: over $100 \mathrm{M}$ website views per year

At BMC, research is always in progress.

Learn more biomedcentral.com/submissions 